\title{
Smart Door Access using Facial Recognition
}

\author{
Amit Deshwal, Mohnish Chandiramani, Umesh Jagtap, Prof. Amruta Surana
}

\author{
Department of Computer Engineering, PCET'S-NMIET, Talegaon, Maharashtra, India
}

\begin{abstract}
Face is a complex multidimensional visual model and developing a computational model for face recognition is difficult. The paper presents a methodology for face recognition based on information theory approach of coding and decoding the face image. The goal is to implement the system (model) for a particular face and distinguish it from a large number of stored faces with some real-time variations as well. The Eigenface approach uses Principal Component Analysis (PCA) algorithm for the recognition of the images. It gives us efficient way to find the lower dimensional space. In today's world, face recognition is an important part for the purpose of security and surveillance. Hence there is a need for an efficient and cost effective system. Our goal is to explore the feasibility of implementing Raspberry Pi based face recognition system using conventional face detection and recognition techniques such as Haar detection and PCA. This paper aims at taking face recognition to a level in which the system can replace the use of passwords and RF I-Cards for access to high security systems and buildings. With the use of the Raspberry Pi kit, we aim at making the system cost effective and easy to use, with high performance.
\end{abstract}

KEYWORDS: Face recognition; face detection; Principal Component Analysis, Raspberry Pi, Eigen faces; Eigen values; Eigenvector

\section{INTRODUCTION}

The information age is quickly revolutionizing the way transactions are completed. There is a need for a faster and accurate user identification and authentication method. Face recognition has become one of the most important user identification methods. Literature survey statistics shows that research work in face recognition system is in its booming era, and in the past forty years, the research in this field has increased exponentially. Face recognition technology emulates the capabilities of human eyes to detect faces. This is done by smart computing that creates "face bunch" that consists of 70 nodal points. Features are extracted from the face and saved as templates. These templates are compared to the face detected. For this research, we interfaced an LCD, Camera and a Motor to the Raspberry Pi board. We have made a real time application, which compares the scans to records stored in the Raspberry $\mathrm{Pi}$ which in turn is used as a gate pass, wherein the name of the detected person is displayed on LCD and the motor will rotate indicating opening and closing of the gate. The eigenfaces may be considered as a set of features which characterize the global variation among face images. Then each face image is approximated using a subset of the eigenfaces, those associated with the largest eigenvalues. These features account for the most variance in the training set. In the language of information theory, we want to extract the relevant information in face image, encode it as efficiently as possible, and compare one face with a database of models encoded similarly. A simple approach to extracting the information contained in an image is to somehow capture the variations in a collection of face images, independently encode and compare individual face images.

\section{MOTIVATION}

Being a student of engineering, we have had experience in programming but we haven't had experience in working real time solutions using our knowledge of programming. We think people shouldn't have to settle when it comes to home security. Technology can deliver both quality and convenience in door lock hardware. And the strongest insights come from understanding the people who use it. The need for using keys to unlock a door can be completely eliminated. We can Replace PC with low-cost processors which make the administrators to get parameters of all the remote devices and to send control information to all the equipment at all the time. It has countless applications based on different environments and scenarios

\section{LITERATURE SURVEY}

Paper Name: Face Recognition System Using Eigen faces. Authors: Ishita Gupta, Varsha Patil.

Year:2014.

Description: Face Recognition technology emulates the capabilities of human eye to detect faces. This is done by smart computing that creates "face bunch" that consists of 70 nodal points. Features are extracted from the face and saved as templates. These templates are compared to the face detected.

Paper Name: An extended set of Haar-like features for rapid project detection.

Authors: Hteik Htar Lwin, Aung Soe Khaing.

Year: 2016.

Description: This paper is a step towards developing a face recognition system which can recognize static images and can be modified to work with dynamic images. In that case the dynamic images received from the camera can first be converted into the static ones and then the same procedure can be applied on them.

Project Name: Face Recognition using Eigenfaces \& Distance Classifiers.

Authors: AV Krishna Rao Padlaya, K Sai Prasanth D Neeraja. Year: 2009.

Description: Facial Recognition is a computer application composes for complex algorithms that use mathematical and metrical techniques, these get the image in raster mode (digital format) and then process and compare pixel by pixel using different methods for obtain a faster and reliable

Paper Name: A Review Paper on Raspberry Pi Authors: Pritish Sachdeva and Shrutik Katchii Year: 2014. 
Description: The Raspberry Pi is a very powerful computer having the dimensions of a business card. In this paper we review Raspberry Pi whose popularity has taken the learning process and application of ideas to whole new level. We also discuss some of the best projects that have come up so far.

\section{EXISTING SYSTEM}

Previous door unlocking system was manual and required human interaction. As we are achieving automation in every field of work so it's a necessity nowadays to reduce the human efforts.

\section{$>$ Disadvantage}

Existing system was not automated \& it requires human efforts.

\section{ARCHITECTURE OF PROPOSED SYSTEM}

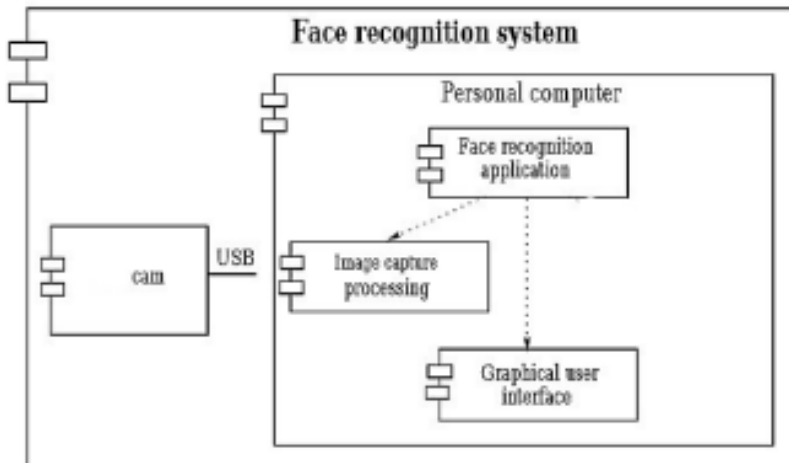

\section{PROPOSED SYSTEM}

The main purpose of the system is to detect and recognize the human face using the OpenCV library on the Raspberry Pi platform. The system will use this data to unlock the door automatically using a servo motor and a Lock-style solenoid switch. If an unrecognized face is detected, then the admin will be notified.

\section{Advantages}

1. Security levels will be significantly improved.
2. The integration process is easy and flawless.

3. High accuracy allows avoiding false identification.

4. Facial Recognition System is fully automated.

\section{CONCLUSION}

In this study, we used the eigenfaces to represent the features vectors for human faces. The features are extracted from the original image to represents unique identity used as inputs to the neural network to measure similarity in classification and recognition. The eigenfaces has proven the capability to provide the significant features and reduces the input size for neural network. Thus, the network speed for recognition is increased.

\section{REFERENCES}

[1] A. M. Patil, Dr. Satish R. Kolhe, Dr. Pradeep M. Patil (2009), "Face Recognition by PCA Technique", Second International Conference on Emerging Trends in Engineering and Technology, ICETET-09

[2] M. A. Turk and A. P. Pentland,"Face recognition using eigenfaces" Proceedings. 1991 IEEE Computer Society Conference on Computer Vision and Pattern Recognition, Maui, HI, 1991, pp. 586-591.

[3] W.Zhao, R. Chellappa, P. J. Phillips, A. Rosenfeld, "Face Recognition: A Literature Survey", ACM Computing Surveys, Vol. 35, No. 4, December 2003, pp. 399-458.

[4] "Face Recognition using Eigenfaces and Distance Classifiers: A Tutorial" by Shubhendu Trivedi, February $11,2009$.

[5] Y. C. Huei,"Benefits and introduction to python programming for freshmore students using inexpensive robots," 2014 IEEE International Conference on Teaching, Assessment and Learning for Engineering (TALE), Wellington, 2014, pp. 12-17.

[6] "Face Recognition using Eigen Faces and Artificial Neural Network" by Mayank Agarwal, Nikunj Jain, Mr. Manish Kumar and Himanshu Agrawal. International Journal of Computer Theory and Engineering, Vol. 2, No. 4, August, 2010, 1793-8201. 